\title{
Indigenous Design for Automatic Testing of Tensile Strength Using Graphical User Interface
}

\author{
Rafay Ali, Faraz Junejo, Rafey Imtiaz and Usama Sultan Shamsi \\ Shaheed Zulfikar Ali Bhutto Institute of Science and Technology, Mechatronics Engineering Department, Karachi, Pakistan
}

\begin{abstract}
Tensile Testing is a fundamental material test to measure the tenacity and tensile strength. Tensile strength means ability to take tensile stress. This Universal Testing Machine is designed using Dual Cylinder Technique in order to comply with the maximun load (tensile force) with the reduction of minimum physical effort and minimized losses.It is to provide material testing opportunity to the students of different institutions, locally and globally, at lowest price; so that they can have a comprehensive understanding of the testing procedures and examining material properties on practical grounds with a minimum expenditure. The testing mechanism is automated along with the inclusion of all necessary parameters and the movement of the members. Specimens of different engineering materials were used to carry out the test and their mechanical strength was tested and compared with the actual values.
\end{abstract}

\section{Introduction}

New methodologies to measure the physical properties of thin films are currently required. Particularly, reported mechanical properties of materials at these dimensions are currently controversial in the scientific literature. Thus, it is necessary to propose techniques for determining mechanical properties of thin films, such as elastic modulus, Poison's ratio and strength. Properties of materials at micro and nanoscale are of considerable interest because of the unique properties associated with small volumes.

Tensile testing is an effective way to investigate the mechanical properties of materials and it is a wellestablished technique for bulk sample characterization. However, tensile testing is not easily implemented for micro and nano-structured materials due to the small dimensions of the specimen. At present, there exist the testing machines for materials, are expensive and its flexibility to make modifications is limited.

An important feature of this device is the simplicity to exchange components according to the user requirements: the low cost, low machine compliance and the high resolution obtained. This machine can also be adapted for compression testing with appropriate samples and grips.

These universal testing machines are specifically designed for demanding testing situations and are suitable for applications from all fields, whether for quality control or for research projects. All Universal Testing Machines can be modified as required to suit individual requirements.

Commercial testing machines with the required capabilities are available, however they are marginally costly. The tester will need to be assembled for a fraction of the cost of an off the shelf tester while retaining the same capabilities as commercially available systems [1].
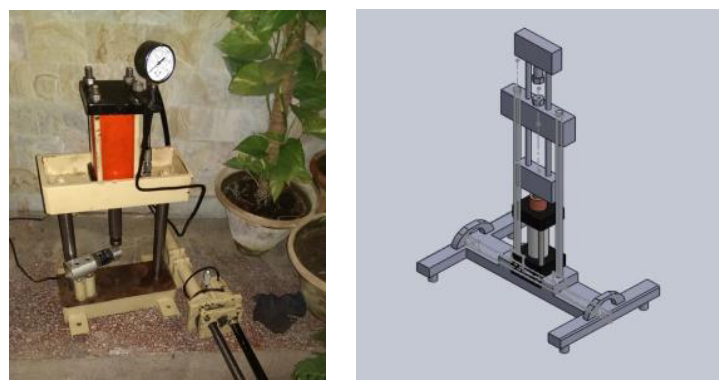

Figure 1. Real design solid work concept

\section{Materials \& method}

Fig. 1 presents a schematic of the Mechanical support base which is assembled with the cross member shown. The cross-member slides through the cross-head by means of the guide rods. The structural components of the testing system are used to subject material specimens to tensile or compressive forces from which we can derive very useful material-specific properties in the observations known as a stress strain plot. While the motion control and data acquisition is done through the computer. A servo motor provides the necessary torque to turn the master cylinder of the hydraulic actuator [2]. The overall electrical system consists of a servo motor and driver circuit, an optical encoder, an amplifier, and a controller card. The function of the encoder is to measure the rotation of the servomotor. The controller and a PCbus plug-in interface card connect the computer to the mechanical testing system. It is planned to connect 
Analog inputs such as load cell, displacement, and strain gauge to this card using the auxiliary channels for data acquisition [3].

The machine is capable of applying a tensile force of $5 \mathrm{KN}$ (limiting force) and is operated using hydraulic oil. The test process involves placing the test specimen in the testing machine and slowly extending it until it fractures. During this process, the elongation of the gauge section is recorded against the applied force. The data is manipulated so that it is not specific to the geometry of the test sample. The elongation measurement is used to calculate the engineering strain, $\epsilon$, using the following equation (Eq. 1):

$$
\epsilon=\delta \mathrm{L} / \mathrm{L} \_0=\left(\mathrm{L}-\mathrm{L} \_0\right) / \mathrm{L} \_0
$$

where $\delta \mathrm{L}$ is the change in gauge length, $\mathrm{L}_{-} 0$ is the initial gauge length, and $\mathrm{L}$ is the final length.

The force measurement is used to calculate the engineering stress, $\sigma$, using the following equation (Eq. 2):

$$
\sigma=F / A
$$

where $\mathrm{F}$ is the tensile force and $\mathrm{A}$ is the nominal crosssectional area of the specimen. The machine does these calculations as the force increases, so that the data points can be graphed into a stress-strain curve.

The shoulders of the test specimen can be manufactured in various ways to mate to various grips in the testing machine. Each system has advantages and disadvantages; for example, shoulders designed for serrated grips are easy and cheap to manufacture, but the alignment of the specimen is dependent on the skill of the technician. On the other hand, a pinned grip assures good alignment. Threaded shoulders and grips also assure good alignment, but the technician must know to thread each shoulder into the grip at least one diameter's length, otherwise the threads can strip before the specimen fractures [4].

In large castings and forgings it is common to add extra material, which is designed to be removed from the casting so that test specimens can be made from it. These specimens may not be exact representation of the whole workpiece because the grain structure may be different throughout. In smaller workpieces or when critical parts of the casting must be tested, a workpiece may be sacrificed to make the test specimens. For workpieces that are machined from bar stock, the test specimen can be made from the same piece as the bar stock [4].
Alignment of the test specimen in the testing machine is critical, because if the specimen is misaligned, either at an angle or offset to one side, the machine will exert a bending force on the specimen. This is especially bad for brittle materials, because it will dramatically skew the results [5]. This situation can be minimized by using spherical seats or U-joints between the grips and the test machine. If the initial portion of the stress-strain curve is curved and not linear, it indicates the specimen is misaligned in the testing machine. The strain measurements are most commonly measured with an extensometer, but strain gauges are also frequently used on small test specimen or when Poisson's ratio is being measured.

\section{Implementation}

Working Principle. The working principle of our project includes the mechanism of rotating the screw jack mechanically using the handle provided with a key so that it remains fixed within. When the screwjack rotates, the pressure is exerted though a master cylinder, attached directly with the screw jack, and the force is transmitted through the cylinder to the crosshead.

The force member (crosshead) moves in the upward direction exerting the force on the load-cell and ultimately exerting the tensile force on the specimen attached. The force is continuously exerted till the specimen breaks and fracture strength is achieved. This stepwise increment of the force and its effects on the specimen are recorded as a data set of values and computation is being simultaneously done on every data set using our created Graphics User Interface (GUI).

The set of observations are recorded in tabular form and a stress-strain curve is plotted with the help of these values using a Graphics User Interface (GUI). The machine must have the proper capabilities for the test specimen being tested. There are four main parameters: force capacity, speed, precision and accuracy. Force capacity refers to the fact that the machine must be able to generate enough force to fracture the specimen. The machine must be able to apply the force quickly or slowly enough to properly mimic the actual application. Finally, the machine must be able to accurately and precisely measure the gauge length and forces applied; for instance, a large machine that is designed to measure long elongations may not work with a brittle material that experiences short elongations prior to fracturing[6].

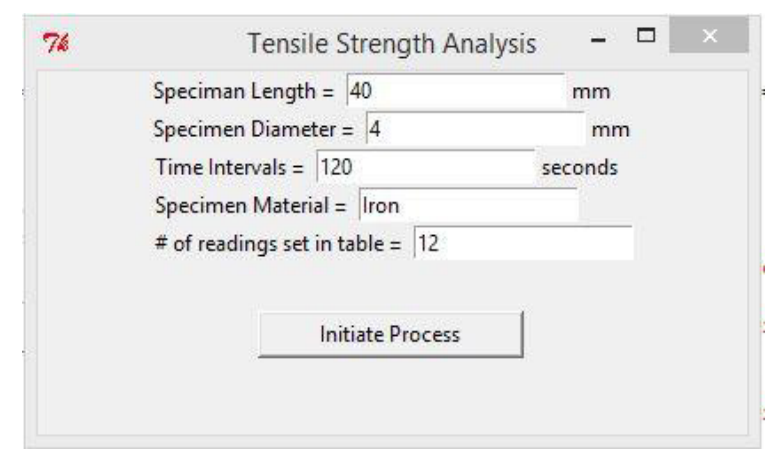




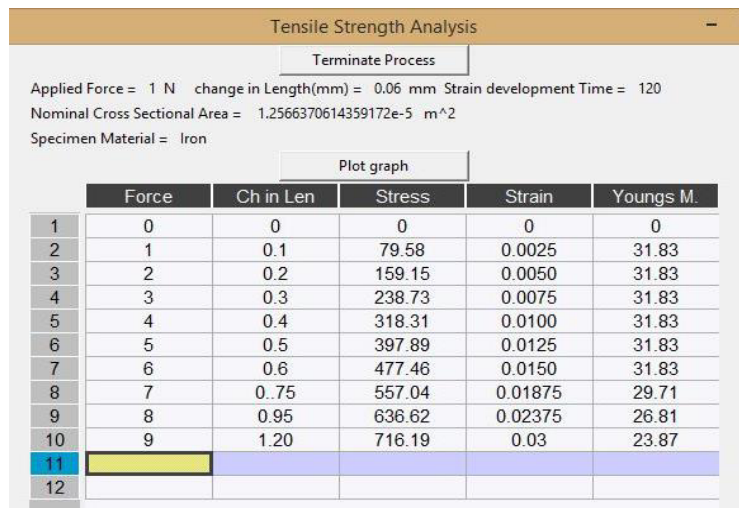

Figure 2. Graphics user interface (Specimen details)

\section{Analysis}

Before initiating the tensile test, user-defines important experiment parameters in relevant fields.

This prompts a new window tabulating the readings based on first screen. As we see initially, the fields are empty but they will populate after each time interval passes. These are processed readings gained from the Loadcell and LVDT sensors. The Young's modulus is then calculated from the stress and strain values. The process is followed by the set of observations taken with constant increment in the force applied on the attached specimen as seen in Fig. 2.
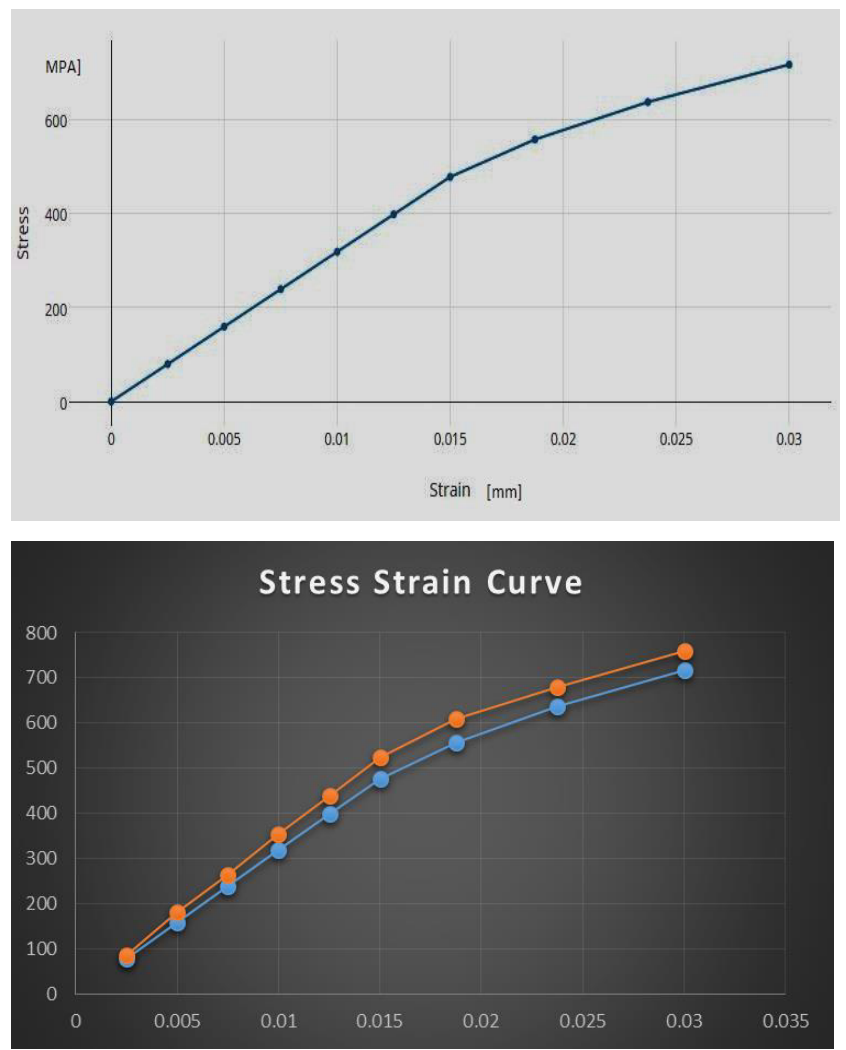

Figure 3. Stress strain curve (Experimented VS Actual curve)

Through these set of observations the stress strain curve is plotted. The graph in Fig. 3 below shows the comparison between the experimented values (blue) and the tested values (orange) as plotted.

The percentage error between the values can be calculated by using the following formula
$\%$ error $=\mid($ experimentalvalue-theoretical value $) /($ theoretical value $)$

$\%$ error $=|(79.85-85) /(85)|$

$\%$ error $=6.05 \%$

\section{Results}

The performance of this Universal Testing Machine indicates that it is appropriate to obtain reliable mechanical properties of compliant materials in thin and soft materials. The testing phase was completed for elastic deformation of the steel specimen by the end of the project timeline. Of these, Iron, Steel and Aluminum specimen were acquired. For a grade450 steel specimen, we can read off yield strength value as $450 \mathrm{Mpa}$. If we observe the graph generated by the machine we see that nonlinearity occurs at approximately 480 Mpa. Furthermore, the obtained observation set of values is compared to the actual available values and the error is calculated. The error is found to be $6 \%$.However, the environment temperature, purity of material specimen and other factors could lead to slight deviation and minimizing these factors to controllable extent could increase the machine's performance and optimum results. The standard set of values has been referred from Shigley's, 8th edition [7].

\section{Conclusion}

This Automated UTM is designed to provide a helping hand to the students and personnel associated with the Mechatronics Engineering field and to provide them the better understanding of the UTM machine of international standard. Secondly, provide Pakistan this machine, manufacturing it locally at a very reasonable cost with exceptional functionality and automated mechanism. The stated objective was to design and build a low-cost, compact prototype for conducting axial load testing, should also act as a platform for future expansion, incorporating a modular design and flexible fixture mounting points to allow for the addition of new capabilities as needed. Furthermore, this provides us with an opportunity to be the entrepreneurs with its development to the industrial scale. This will provide a means of employment opportunities involved in the development when taking it to the mass manufacturing level. 
The system was envisioned to provide additional lowend support to the students of our University. Thus, the aim, set to overcome the difficulties faced by the students in understanding the actual concept of behavior (elastic and plastic) exhibited by different engineering materials (ductile and brittle) under the action of applied load along with the integration of different electronic equipment: sensors (loadcell and LVDT), so that they can have a visual representation of the different regions of the material under load. Yet, as the project evolved and the promising prototype that we saw coming together, this project has the potential to provide undergraduate students hands-on experience in setting up and conducting mechanical testing.

\section{References}

1. Theusen, G. J. and Fabrycky, W. J. Engineering Economy. 6th edition. Englewood Cliffs, N. J.: Prentice-Hall. 1984.

2. Juvinall, R. C., and Marshek, K. M., 1991. Fundamentals of machine component design, John Wiley \& Sons, Inc., NY, pp. 349.

3. Information on http://library.utm.utoronto.ca/

4. Standard test method for tensile properties of plastics, ASTM International

5. Wang, L., Lau, J., Thomas, E. L., and Boyce, M. C., 2011.Co-continuous composite materials for stiffness, strength, and energy dissipation, Advanced Materials 23(13), April, pp. 1524-1529.

6. Billings. S. A. Identification of Nonlinear Systems: A Survey. Proc. Instn Electr. Engrs, Part D, 127(6): 272-284, (1980).

7. Budynas, R. G., and Nisbett, J., K., 2008. Shigley's Mechanical Engineering Design, 8th edition. McGraw-Hill, NY, pp. 1006. 